\title{
La relación parental y el desarrollo de la lectoescritura en estudiantes de la escuela de educación básica "Fe y Alegría" del cantón Ambato
}

The parental relationship and the development of literacy in students at the basic education school "Fe y Alegría" of the canton Ambato

1 Karen Estefanía Núñez Pilco https://orcid.org/0000-0002-4094-4140 Universidad Técnica de Ambato, Facultad de Ciencias Humanas y de la Educación, Carrera de Psicopedagogía. Ambato, Ecuador. knunez3648@uta.edu.ec

2 Verónica del Carmen Llerena Poveda iD https://orcid.org/0000-0002-0077-6322 Universidad Técnica de Ambato, Facultad de Ciencias Humanas y de la Educación, Carrera de Psicopedagogía. Ambato, Ecuador. veronicadllerena@uta.edu.ec

3 Carolina Elizabeth Manzano Vinueza (iD) https://orcid.org/0000-0001-5421-4002 Universidad Técnica de Ambato, Facultad de Ciencias de la Educación, Carrera de Psicopedagogía. Ambato, Ecuador. carolinaemanzanov@uta.edu.ec

Artículo de Investigación Científica y Tecnológica Enviado: 24/12/2021 Revisado: 29/12/2021 Aceptado: $12 / 01 / 2022$ Publicado:08/03/2023 DOI: https://doi.org/10.33262/concienciadigital.v6i1.4.2008

Cítese:

Núñez Pilco, K. E., Llerena Poveda, V. del C., \& Manzano Vinueza, C. E. (2023). La relación parental y el desarrollo de la lectoescritura en estudiantes de la escuela de educación básica "Fe y Alegría" del cantón Ambato . ConcienciaDigital, 6(1.4), 456-473. https://doi.org/10.33262/concienciadigital.v6i1.4.2008

CONCIENCIA DIGITAL, es una Revista Multidisciplinar, Trimestral, que se publicará en soporte electrónico tiene como misión contribuir a la formación de profesionales competentes con visión humanística y crítica que sean capaces de exponer sus resultados investigativos y científicos en la misma medida que se promueva mediante su intervención cambios positivos en la sociedad. https://concienciadigital.org .

La revista es editada por la Editorial Ciencia Digital (Editorial de prestigio registrada en la Cámara Ecuatoriana de Libro con No de Afiliación 663) www.celibro.org.ec 


\section{Palabras}

claves:

relación

parental, lectoescritura, docente tutor, desarrollo

\section{Keywords:}

parental relationship, literacy, teacher tutor, development

\section{Resumen}

Introducción. Debido al cambio y la modalidad que se emplea actualmente los padres de familia han tomado el papel protagónico en la educación de los hijos siendo ellos quienes juegan un papel de docente tutor dentro de los hogares. Objetivo. Establecer la influencia de la relación parental en el desarrollo de la Lectoescritura en estudiantes de tercer año de E.G.B de la escuela de educación básica "Fe y Alegría" del cantón Ambato. Metodología. Para el cumplimiento del presente trabajo de investigación se consideró información bibliográfica para apoyar a las variables planteadas. Así tiene un enfoque cualitativo y cuantitativo con un nivel descriptivo y correlacional; además para la recolección de información se utilizó un cuestionario para padres de familia validado por expertos y la aplicación de la prueba LEE, la población que se consideró fueron los estudiantes de Tercer año de E.G.B. paralelos “A y B" con un total de 46 estudiantes. Resultados. Los resultados demuestran que la relación parental influye en el desarrollo de la lectoescritura, específicamente si los padres realizan actividades que promuevan e incentiven a desarrollar la misma habilidad; los datos fueron sometidos al software SPS-S para la validación de hipótesis utilizando el estadígrafo las tablas cruzadas de Tau-B de Kendall. Conclusión. Se concluyó que los padres de familia quienes realizan un plan para el desarrollo de la lectoescritura logran que sus hijos tengan mayor habilidad a la hora de leer y escribir el cual se evidencia por la calidad y fluidez de este proceso.

\section{Abstract}

Introduction. Due to the change and the modality that is currently used, parents have taken the leading role in the education of their children, being they who play a role of teaching tutor within the homes. Target. To establish the influence of the parental relationship in the development of Literacy in third-year students of E.G.B of the basic education school "Fe y Alegría" of the Ambato canton. Methodology. For the fulfillment of this research work, bibliographic information was thought to support the proposed variables. Thus, it has a qualitative and quantitative approach with a descriptive and correlational level; In addition, for the collection of information, a questionnaire for parents validated by experts and the application of the LEE test was produced, the expected 
population is third-year students of E.G.B. parallels "A and B" with a total of 46 students. Results. The results show that the parental relationship influences the development of literacy, specifically if the parents carry out activities that promote and encourage the development of the same skill; the data were submitted to the SPS$\mathrm{S}$ software for hypothesis validation using Kendall's Tau-B crosstables statistician. Conclusion. It was concluded that parents who made a plan for the development of literacy achieved that their children have greater ability to read and write which is evidenced by the quality and fluency of this process.

\section{Introducción}

Relación Parental. - La familia se define como la unidad básica de la sociedad, por tal motivo el rol de los padres se vuelve indispensable para que el niño empiece una convivencia social; además de ser los encargados del desarrollo de aptitudes y el desarrollo socioafectivo desde los primeros años de vida (Ramírez et al., 2015). La familia juega un rol fundamental en la primera infancia debido a que se encarga de educar e impulsar al infante. Con el tiempo se transforman y se convierten en seres sociales; esto se refleja cuando adquiere experiencias, pensamiento lógico además de valores que son impartidas por la misma familia por ende ayuda a estructurar características propias en el sujeto. En resumen, la familia juega un rol fundamental en la vida del infante al ser el primer acercamiento que tiene con la sociedad brinda las herramientas y pautas necesarias para insertarse y convivir con los demás. La relación contenedora y saludable que brindan los padres es fundamental para incentivar aspectos psicológicos y sociales en el niño (Krumm et al., 2017).

Participación de la familia en la educación. - Razeto (2016), resalta la importancia de la participación de los padres en la escuela ya que potencian las capacidades adaptativas, sociales e inciden en el éxito académico. La participación de los padres para Palomeque et al. (2018). Forma parte complementaria del proceso pedagógico dentro de las unidades educativas, entendiéndose por participación familiar a la forma activa y recíproca entre la relación de la escuela y la familia en el cual cada parte muestra interés, impone un compromiso constante entre las responsabilidades de los actores de este proceso; además dicha participación debe ser libre y respetada de manera recíproca entre sus partes.

Padres y educación en confinamiento. - Díaz Barriga (2020), menciona que hoy en día los progenitores están a cargo de atender la escolaridad de sus hijos; esto ha provocado un aumento en las labores de madres y padres quienes deben centrar su atención en su trabajo, labores domésticas y ahora atender las dudas y preguntas que presentan los educandos durante el proceso de escolarización. En consecuencia, de la pandemia la 
participación constante de los padres durante el proceso de escolarización para Velásquez y Ruidiaz (2021), es evidente que se entrelazaron una constante cercanía entre padres, docentes, alumnos para enfrentarse a los retos de la nueva modalidad de estudio; así se alcanzarán los objetivos planteados dentro del proceso escolar.

Lectoescritura. - La lectoescritura para Gil (2019), es la capacidad del ser humano para identificar la representación gráfica de los sonidos del habla y es el proceso de entender que los símbolos alfabéticos pertenecen a los sonidos que se perciben o se emiten. El aprendizaje de la lectura y la escritura permiten a los niños dialogar sobre sus realidades, crear nuevas expectativas y finalmente construir significados de forma activa. El proceso lectoescritor empieza desde los primeros años y al llegar a la escuela trae consigo herramientas intelectuales, afectivas, comunicativas que se ha formado por medio de las experiencias que su contexto familiar y social le han ofrecido (Valenciano-Canet, 2019).

Importancia de la lectoescritura. - La lectoescritura es un elemento fundamental en el proceso de aprendizaje ya que garantizará el óptimo rendimiento académico; además sirve para generar aprendizajes significativos a futuro por lo cual es necesario el desarrollo del: lenguaje oral, precisión en la lectura de palabras, velocidad o fluidez, comprensión, corrección ortográfica, gramatical y de la puntuación, claridad u organización de la expresión escrita. Por dichos factores antes mencionados el proceso de lectoescritura se vuelve indispensable en la vida de cada individuo (Esquivel \& Aldrete, 2019).

Conciencia Fonológica. - Para el desarrollo adecuado de la lectura y escritura es indispensable que el niño posea una conciencia fonológica. Para Gutiérrez y Díez (2018), la conciencia fonológica es la habilidad que permite acceder a la estructura de la lengua oral y ser consciente de los segmentos fonológicos de las palabras. Carpio (2017), resalta la importancia de la conciencia fonológica a la hora de leer ya que se habla sobre la habilidad del niño al momento de identificar, separar y combinar de forma voluntaria las unidades sub-léxicas, además de ayudar al desarrollo de la lectura se vincula directamente para producir la escritura, la adquisición de estas habilidades apertura a diferenciar entre buenos y malos lectores (Calle et al., 2016).

Lectura. - Gutiérrez (2018), describe a la lectura como el vínculo entre el lenguaje oral y escrito, además resalta la importancia de procesar y entender a cada grafema como un elemento sonoro así darle el significado correspondiente con un sonido; debido a que se basa en una segmentación encadenada del habla. Rosero \& Mieles (2015), mencionan que un adecuado proceso de estimulación lectora fortalece dentro del individuo las áreas comunicativas, cognitivas y creativas, esto le permite dar significados a objetos, dar sentido a algo además permite crear una realidad en base a lo que le rodea. 
Escritura. - Valencia et al. (2019), describe a la escritura como un sistema complejo debido a que implica diversas acciones y operaciones que parten desde el análisis de los sonidos hasta una adecuada decodificación de un fonema, el aprendizaje de la escritura para Silva et al. (2016) es un proceso que describe al lenguaje escrito como un objeto de conocimiento que se relaciona con el medio en donde se desarrolla.

\section{Metodología}

Para el proyecto planteado se desarrolló el siguiente diagrama de flujo de la figura 1, en cual se plantea la forma en la que se llevarán a cabo las actividades.

Primero se realizó una búsqueda bibliográfica que permitió el estudio del arte lo cual ayuda a entender de mejor forma el enfoque del proyecto. Con ello también se consigue determinar cuál será el parámetro de estudio que ira a ser analizado, que en este caso será la comprensión lectora.

Posteriormente se realizó la búsqueda de las pruebas que permitirán la evaluación del parámetro planteado para el estudio del caso y ayudara a determinar los resultados de la investigación. Para lo cual se planteó diversos parámetros que debían cumplir las pruebas para que sirvieran en la obtención de resultados del caso de estudio, los cuales fueron: edad, función, población. La edad es de vital importancia ya que segrega el grupo En el cual será focalizado el análisis del parámetro de estudio. La función permite entender cuál es el enfoque del material de apoyo en el cual se sustenta la investigación. Y por último la población que es determinada por la región y en este caso la unidad educativa en la cual se realizara el estudio.

Si cumple con los parámetros el material de evaluación seguirá para su aplicación en la población analizada, sin embargo, de no ser así se retornará a la búsqueda de otro test que cumpla con los requerimientos anunciados anteriormente.

Para este caso se seleccionaron dos instrumentos que cumplían con los requerimientos de la población de estudio los cuales debían ser test aplicables para una población estudiantil y una encuesta aplicable a los progenitores. Estos fueron:

\section{Test Lee. - Cuestionario para padres}

Una vez el test se apruebe se procede a la aplicación de este en la población de estudio se tabularán los datos con lo cual se podrán analizar y llegar a la conclusión acerca del estudio. 


\section{DDigital}

\section{Figura 1}

Flujograma de la metodología empleada en el proyecto

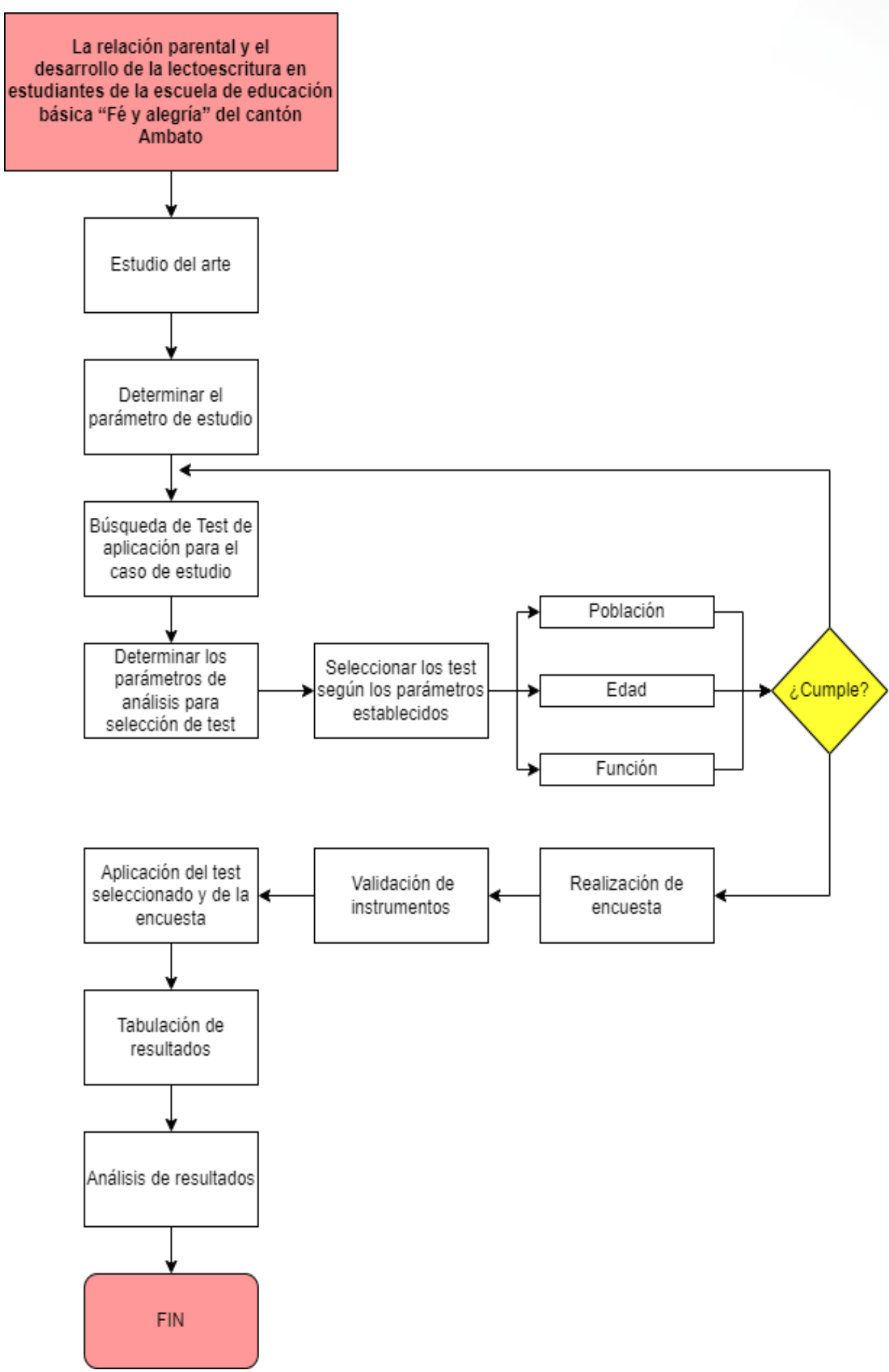

Nota: Elaboración de un diagrama para la investigación. 


\section{Población y Muestra}

La población está constituida por estudiantes de la escuela de educación básica "Fe y Alegría". Para esta investigación se consideró como muestra a 46 estudiantes del tercer año de Educación General Básica.

\section{Tabla1}

\section{Población}

\begin{tabular}{ccr}
\hline Unidades de observación & Frecuencia & Porcentaje \% \\
\hline Estudiantes de 3ro.E.G. B "A" & 25 & $54 \%$ \\
Estudiantes de 3ro.E.G. B "B" & 21 & $46 \%$ \\
Total & 46 & $100 \%$ \\
\hline
\end{tabular}

Fuente: Escuela de educación básica "Fe y Alegría"

Elaborado por: Karen Estefanía Núñez Pilco

Plan de recolección de información

Tabla 2

Plan de recolección de información

\begin{tabular}{|c|c|}
\hline Preguntas básicas & Explicación \\
\hline ¿Para qué? & $\begin{array}{l}\text { Alcanzar los objetivos planteados por la } \\
\text { investigación }\end{array}$ \\
\hline ¿Sobre qué aspectos? & Relación parental y el desarrollo de la lectoescritura \\
\hline ¿Quién? & Karen Estefanía Núñez Pilco \\
\hline ¿A quiénes? & $\begin{array}{l}\text { A los estudiantes de Tercer año de E.G.B y padres } \\
\text { de familia }\end{array}$ \\
\hline ¿Cuándo? & Período académico abril- septiembre 2021 \\
\hline ¿Dónde? & Escuela de Educación Básica "Fe y Alegría" \\
\hline ¿Cuántas veces? & Una vez \\
\hline $\begin{array}{l}\text { ¿Qué técnica de } \\
\text { recolección? }\end{array}$ & Psicométrica y encuesta \\
\hline ¿Con qué? & Test LEE y un cuestionario para padres de familia \\
\hline
\end{tabular}

Fuente: Escuela de educación básica "Fe y Alegría"

Elaborado por: Karen Estefanía Núñez Pilco

\section{Técnicas e instrumentos de recolección de información}

La presente investigación utilizará dos instrumentos para las variables el primero por medio de la técnica psicométrica utilizará como instrumento el test LEE para los estudiantes y un cuestionario diseñado para padres.

El Test LEE. Es una prueba de evaluación para niños de 1ro. a 4to. Año de E.G.B. Se 
encuentra subdivida en siete pruebas de las cuales contempla actividades de lectura de palabras y pseudopalabras, comprensión de frases y textos para evaluar el área lectora. Igualmente, evalúa procedimientos de escritura con las secciones escritura de palabras y pseudopalabras. Su enfoque es instrumental con metodología cuantitativa.

Posibilita comparar el rendimiento de los niños en lectura y escritura obteniendo un perfil en el que se observan fortalezas y debilidades en cada uno de los procesos investigados. El Test LEE está organizado alrededor de los dos grandes componentes de la lectura: el reconocimiento de palabras y la comprensión lectora. La aplicación es de forma individual en un tiempo de 45 minutos.

Su calificación se encuentra en una escala de competente, muy competente y con dificultad. El nivel competente se caracteriza por ser una lectura silábica; es decir pausada y poco fluida, en el nivel muy competente se encuentran características de una lectura fluida y una acertada comprensión de textos finalmente el nivel con dificultad se caracteriza por una lectura deficiente, tardada que presenta omisiones, adiciones de fonemas y grafemas a la hora de leer y escribir.

\section{Formulación de encuesta}

Para la variable independiente se utilizará un cuestionario que consta de 20 preguntas dirigido a padres de familia, en la cual se pretende recolectar información sobre la participación de los progenitores en el desarrollo de la lectoescritura consta de una escala de Likert valorada desde el 1 al 5; siendo uno la puntuación totalmente en desacuerdo, dos en desacuerdo, tres indiferente que se traduce a los participantes que no se encuentran en acuerdo ni en desacuerdo, cuatro de acuerdo y finalmente cinco totalmente de acuerdo.

\section{Validación}

Para la respectiva validación de instrumentos se utilizó dos formas:

Para la primera variable de la presente investigación se utilizó el cuestionario para padres de familia que consiste en 20 preguntas dirigidas a los progenitores, valorada en una escala de likert con una puntuación del uno al cinco; el cual permitirá obtener datos relevantes sobre el rol que el padre de familia representa en el desarrollo lectoescritor del niño.

Este instrumento fue sometido a una validación por expertos, docentes de la carrera de Psicopedagogía, desde su criterio técnico, los profesionales sometieron a revisión cada literal, una vez culminada el análisis respectivo se continuó con la aplicación.

Para la segunda variable se utilizó el test Lee el instrumento que permite evidenciar el desarrollo lectoescritor, posibilita comparar el rendimiento de los niños en lectura y 
escritura obteniendo un perfil en el que se observan fortalezas y debilidades en cada uno de estos procesos, con estos antecedentes el instrumento es aplicable en la investigación.

\section{Resultados}

Se estableció por medio de instrumentos validados como la encuesta para padres de familia y la aplicación del test LEE, la estrecha correlación entre la relación parental y el desarrollo de la Lecto-escritura puesto que se determinó que los progenitores sí influyen en el desarrollo lectoescritor en los estudiantes de la escuela de educación básica "Fe y alegría" del cantón Ambato. Debido a que los resultados estadísticos corroboraron que los padres al aplicar un plan para incentivar actividades en la lectura y escritura tienen un impacto en áreas como la lectura de palabras y comprensión de textos.

\section{Tabla 3}

Tabulación de datos obtenidos a partir de la encuesta realizada a los padres de familia de la muestra correspondiente

\begin{tabular}{|c|c|c|c|c|c|}
\hline ÍTEMS & 1 & 2 & 3 & 4 & 5 \\
\hline ¿Cree que su hijo está aprendiendo mediante la modalidad virtual? & $7 \%$ & $22 \%$ & $30 \%$ & $35 \%$ & $7 \%$ \\
\hline $\begin{array}{l}\text { ¿Su hijo mantiene un área específica de estudio al momento de } \\
\text { recibir clases? }\end{array}$ & $2 \%$ & $9 \%$ & $7 \%$ & $37 \%$ & $46 \%$ \\
\hline ¿Su hijo toma nota durante la clase? & $7 \%$ & $15 \%$ & $11 \%$ & $30 \%$ & $37 \%$ \\
\hline ¿Disfruta compartir tiempo con su hijo? & $0 \%$ & $0 \%$ & $9 \%$ & $13 \%$ & $78 \%$ \\
\hline ¿Motiva usted constantemente a su hijo para alcanzar logros? & $7 \%$ & $2 \%$ & $2 \%$ & $13 \%$ & $76 \%$ \\
\hline $\begin{array}{l}\text { Determina un tiempo en específico para ayudarle a su hijo en las } \\
\text { tareas }\end{array}$ & $17 \%$ & $15 \%$ & $20 \%$ & $26 \%$ & $22 \%$ \\
\hline ¿Considera usted importante ceder responsabilidades a los niños? & $0 \%$ & $2 \%$ & $13 \%$ & $30 \%$ & $54 \%$ \\
\hline $\begin{array}{l}\text { Cuando su hijo se encuentra frustrado ¿Usted brinda soporte en } \\
\text { estas situaciones? }\end{array}$ & $9 \%$ & $7 \%$ & $13 \%$ & $26 \%$ & $46 \%$ \\
\hline Impone castigos a su hijo para que aprenda & $15 \%$ & $13 \%$ & $22 \%$ & $37 \%$ & $13 \%$ \\
\hline $\begin{array}{l}\text { Suele apoyar las decisiones que toma el tutor sobre el } \\
\text { acompañamiento escolar de su hijo }\end{array}$ & $0 \%$ & $7 \%$ & $4 \%$ & $54 \%$ & $35 \%$ \\
\hline $\begin{array}{l}\text { ¿Se preocupa por averiguar cuál es el desempeño escolar de su } \\
\text { hijo? }\end{array}$ & $2 \%$ & $4 \%$ & $4 \%$ & $39 \%$ & $50 \%$ \\
\hline $\begin{array}{l}\text { ¿Mantiene una comunicación constante con el docente respecto a } \\
\text { los avances de su hijo? }\end{array}$ & $2 \%$ & $9 \%$ & $20 \%$ & $33 \%$ & $37 \%$ \\
\hline Su hijo ¿demuestra interés por leer? & $22 \%$ & $26 \%$ & $15 \%$ & $22 \%$ & $15 \%$ \\
\hline $\begin{array}{l}\text { Dentro de su hogar ¿Realiza actividades que promuevan la } \\
\text { Lectoescritura? }\end{array}$ & $9 \%$ & $30 \%$ & $20 \%$ & $20 \%$ & $21 \%$ \\
\hline ¿Su hijo lee y escribe con facilidad? & $15 \%$ & $37 \%$ & $4 \%$ & $35 \%$ & $9 \%$ \\
\hline
\end{tabular}




\section{Tabla 3}

Tabulación de datos obtenidos a partir de la encuesta realizada a los padres de familia de la muestra correspondiente (continuación)

\begin{tabular}{|c|c|c|c|c|c|}
\hline ÍTEMS & 1 & 2 & 3 & 4 & 5 \\
\hline $\begin{array}{l}\text { ¿Considera que el docente utiliza herramientas innovadoras } \\
\text { para el desarrollo de la lectoescritura? }\end{array}$ & $7 \%$ & $22 \%$ & $7 \%$ & $35 \%$ & $30 \%$ \\
\hline $\begin{array}{l}\text { Actualmente usted juega el papel de docente tutor ¿Ha } \\
\text { aplicado algún plan para incentivar la Lectoescritura en su } \\
\text { hijo? }\end{array}$ & $9 \%$ & $33 \%$ & $17 \%$ & $30 \%$ & $11 \%$ \\
\hline $\begin{array}{l}\text { Considera usted importante el uso de cuentos, fábulas, } \\
\text { leyendas durante el proceso de aprendizaje lectoescritor }\end{array}$ & $0 \%$ & $0 \%$ & $2 \%$ & $24 \%$ & $74 \%$ \\
\hline Considera usted que la lectura de su hijo es deficiente & $20 \%$ & $15 \%$ & $15 \%$ & $39 \%$ & $11 \%$ \\
\hline $\begin{array}{l}\text { En la educación actual ¿Considera usted que la relación y el } \\
\text { apoyo entre padres e hijos es importante para desarrollar } \\
\text { habilidades en la lectura y escritura? }\end{array}$ & $0 \%$ & $0 \%$ & $0 \%$ & $26 \%$ & $74 \%$ \\
\hline
\end{tabular}

Fuente: Escuela de educación básica "Fe y Alegría" Elaborado por: Karen Estefanía Núñez Pilco

Análisis de los resultados obtenidos de la aplicación del teste de Lee

Los resultados sobre el desarrollo de la lectoescritura de los estudiantes de tercer año de E.G.B, se obtuvieron mediante la aplicación del test Lee, este instrumento se divide en siete subpruebas que son: lectura de palabras, lectura de pseudopalabras, compresión y frases, prosodia, comprensión de textos, escritura de palabras y escritura de pseudopalabras.

\section{Lectura de palabras}

\section{Tabla 4}

Test de Lee (Lectura de Palabras)

\begin{tabular}{lcc}
\hline \multicolumn{1}{c}{ NIVEL } & FRECUENCIA & PORCENTAJE \\
\hline Muy competente & 4 & $9 \%$ \\
Competente & 14 & $30 \%$ \\
Con dificultad & 28 & $61 \%$ \\
Total & 46 & $100 \%$ \\
\hline
\end{tabular}

Fuente: Escuela de educación básica "Fe y Alegría"

Elaborado por: Karen Estefanía Núñez Pilco

\section{Análisis}

Con la aplicación respectiva del test se obtiene que del 100\% de los alumnos, el $61 \%$ que representa a 28 alumnos se encuentran en el nivel con dificultad, el $30 \%$ conformado por 
14 alumnos según el instrumento se encuentra en el nivel competente, y solo el $9 \%$ que representa a 4 estudiantes se encuentran en el nivel muy competente.

\section{Interpretación}

Por lo tanto, la mayor parte de estudiantes representados por el $61 \%$ se encuentra en el nivel con dificultad al momento de leer palabras, esta subprueba consiste en presentar una serie de palabras en la cual el niño debe leer esto permite conocer los procesos léxicos. La característica principal del nivel con dificultad es: una lectura deficiente, silábica es decir pausada una lectura en la cual el niño separa a la palabra por sílabas y poco fluida la cual presenta errores a la hora de decodificar los grafemas, el número de estudiantes que demuestran una lectura competente alcanzan un nivel medio y están representados por el $30 \%$ finalmente el nivel muy competente correspondiendo al $9 \%$ cumplen con una lectura fluida esta se caracteriza por no cometer errores a la hora de reconocer y decodificar palabras siendo el menor porcentaje.

\section{Lectura de pseudopalabras}

Tabla 5

Test de Lee (Lectura de Pseudopalabras)

\begin{tabular}{lcc}
\hline \multicolumn{1}{c}{ NIVEL } & FRECUENCIA & PORCENTAJE \\
\hline Muy competente & 7 & $15 \%$ \\
Competente & 18 & $39 \%$ \\
Con dificultad & 21 & $46 \%$ \\
Total & 46 & $100 \%$ \\
\hline
\end{tabular}

Fuente: Escuela de educación básica "Fe y Alegría"

Elaborado por: Karen Estefanía Núñez Pilco

\section{Análisis}

Con la aplicación respectiva del test se obtiene que del 100\% de los alumnos, el $15 \%$ que representa a 7 alumnos se encuentran en el nivel muy competente, el 39\% conformado por 18 alumnos según el instrumento se encuentra en competente, y el $46 \%$ que representa a 21 estudiantes se encuentran en el nivel con dificultad.

\section{Interpretación}

Por lo tanto, la característica principal de este nivel es la dificultad y la complejidad un tanto elevada de las palabras; un mayor porcentaje representado al $46 \%$ de la población se encuentra en el nivel con dificultad: en este nivel los niños demostraron una lectura pausada, además de que separaban las palabras en sílabas es decir presentaban una lectura silábica; sin embargo existe un porcentaje medio representado por el 39\% de estudiantes que lograron alcanzar el nivel competente, finalmente en el nivel muy competente se encuentra el menor porcentaje representado por el 15\% quiénes dominaron esta sección. 
Comprensión de palabras y frases

\section{Tabla 6}

Test de Lee (Comprensión de palabras y frases)

\begin{tabular}{ccc}
\hline NIVEL & FRECUENCIA & PORCENTAJE \\
\hline Muy competente & 6 & $13 \%$ \\
Competente & 19 & $41 \%$ \\
Con dificultad & 21 & $46 \%$ \\
Total & 46 & $100 \%$ \\
\hline
\end{tabular}

Fuente: Escuela de educación básica "Fe y Alegría"

Elaborado por: Karen Estefanía Núñez Pilco

\section{Análisis}

Con la aplicación respectiva del test se obtiene que del 100\% de los alumnos, el 13\% que representa a 6 alumnos se encuentran en el nivel muy competente, el $41 \%$ conformado por 19 alumnos según el instrumento se encuentra en competente, y el $46 \%$ que representa a 21 estudiantes se encuentran en el nivel con dificultad.

\section{Interpretación}

La subprueba de comprensión de palabras y frases pretende investigar los procesos sintácticos y semánticos de los estudiantes.

Por lo tanto, se puede establecer que el mayor porcentaje de la población evaluada correspondiente al $46 \%$ se mantiene en el nivel con dificultad es decir los niños muestran problemas en la comprensión de palabras y frases por lo cual presentan dificultades para decodificar los procesos léxicos y semánticos de cada uno de las actividades; un porcentaje medio representando al $41 \%$ de la población se establecen en el nivel competente finalmente en el nivel muy competente ocupa el menor porcentaje con un $13 \%$ de la población que domina esta área.

\section{Prosodia}

Tabla 7

Test de Lee (Prosodia)

\begin{tabular}{lcc}
\hline \multicolumn{1}{c}{ NIVEL } & FRECUENCIA & PORCENTAJE \\
\hline Muy competente & 7 & $15 \%$ \\
Competente & 15 & $33 \%$ \\
Con dificultad & 24 & $52 \%$ \\
Total & 46 & $100 \%$ \\
\hline
\end{tabular}

Fuente: Escuela de educación básica "Fe y Alegría"

Elaborado por: Karen Estefanía Núñez Pilco 
Análisis

Con la aplicación respectiva del test se obtiene que del 100\% de los alumnos, el 15\% que representa a 7 alumnos se encuentran en el nivel muy competente, el 33\% conformado por 15 alumnos según el instrumento se encuentra en competente, y el 52\% que representa a 24 estudiantes se encuentran en el nivel con dificultad.

\section{Interpretación}

La característica principal de esta subprueba es evaluar el nivel sintáctico de las oraciones además del uso de los signos de puntuación y como lo interpretan.

Por lo tanto, se puede establecer que el mayor porcentaje de la población evaluada se mantiene en el nivel con dificultad representando al 52\%, en esta sección los estudiantes presentaron dificultades en dar un significado a las frases y a la utilización de diferentes signos de interrogación en la mayoría de las oraciones. Sin embargo, existen un porcentaje medio el cual representa al $33 \%$ de los estudiantes que se encuentran en el nivel competente finalmente el menor porcentaje representado por el $15 \%$ de los estudiantes lograron dominar esta sección.

Comprensión de textos

\section{Tabla 8}

Test de Lee (Comprensión de textos)

\begin{tabular}{lcc}
\hline \multicolumn{1}{c}{ NIVEL } & FRECUENCIA & PORCENTAJE \\
\hline Muy competente & 7 & $15 \%$ \\
Competente & 20 & $43 \%$ \\
Con dificultad & 19 & $41 \%$ \\
Total & 46 & $100 \%$ \\
\hline
\end{tabular}

Fuente: Escuela de educación básica "Fe y Alegría"

Elaborado por: Karen Estefanía Núñez Pilco

\section{Análisis}

Con la aplicación respectiva del test se obtiene que del 100\% de los alumnos, el 15\% que representa a 7 alumnos se encuentran en el nivel muy competente, el $43 \%$ conformado por 20 alumnos según el instrumento se encuentra en competente, y el $41 \%$ que representa a 19 alumnos se encuentran en el nivel con dificultad.

\section{Interpretación}

Por lo tanto, se puede establecer que el mayor porcentaje de la población evaluada se mantiene en el nivel competente representado por el $43 \%$ la característica principal de esta subprueba es presentar diversos textos y responder de forma acertada a las preguntas esto permite indagar respecto a los procesos semánticos y la lectura compresiva, por otro 
lado un porcentaje medio representado por el $41 \%$ se encuentra en el nivel con dificultad la población tuvo problemas a la hora de resolver esta sección. Finalmente, el menor porcentaje se sitúa en el nivel muy competente representado por el $15 \%$ de la población debido a que respondieron de forma acertada a las preguntas realizadas sobre cada texto.

\section{Escritura de palabras}

Tabla 9

Test de Lee (Escritura de palabras)

\begin{tabular}{lcc}
\hline \multicolumn{1}{c}{ NIVEL } & FRECUENCIA & PORCENTAJE \\
\hline Muy competente & 4 & $9 \%$ \\
Competente & 23 & $50 \%$ \\
Con dificultad & 19 & $41 \%$ \\
Total & 46 & $100 \%$ \\
\hline
\end{tabular}

Fuente: Escuela de educación básica "Fe y Alegría"

Elaborado por: Karen Estefanía Núñez Pilco

\section{Análisis}

Con la aplicación respectiva del test se obtiene que del 100\% de los alumnos, el 9\% que representa a 4 alumnos se encuentran en el nivel muy competente, el 50\% conformado por 23 alumnos según el instrumento se encuentra en competente, y el $41 \%$ que representa a 19 alumnos se encuentran en el nivel con dificultad.

\section{Interpretación}

Por lo tanto, se puede establecer que el mayor porcentaje de la población evaluada se mantiene en el nivel competente representado por el $50 \%$ de los estudiantes, un porcentaje medio representado por el $41 \%$ se establece en el nivel con dificultad. Esta subprueba pretende indagar respecto a los procesos fonológicos y ortográficos de los estudiantes, se encuentran en el nivel con dificultad por presentar omisiones, adiciones al momento de escribir. Finalmente, el menor porcentaje representado por el $9 \%$ de estudiantes se sitúan en el nivel muy competente es decir la población alcanza y domina esta subprueba.

Escritura de Pseudopalabras

Tabla 10

Test de Lee (Escritura de Pseudopalabras)

\begin{tabular}{lcc}
\hline \multicolumn{1}{c}{ NIVEL } & FRECUENCIA & PORCENTAJE \\
\hline Muy competente & 5 & $11 \%$ \\
Competente & 19 & $41 \%$ \\
Con dificultad & 22 & $48 \%$ \\
Total & 46 & $100 \%$ \\
\hline
\end{tabular}

Fuente: Escuela de educación básica "Fe y Alegría"

Elaborado por: Karen Estefanía Núñez Pilco 


\section{Análisis}

Con la aplicación respectiva del test se obtiene que del 100\% de los alumnos, el $11 \%$ que representa a 5 alumnos se encuentran en el nivel muy competente, el $41 \%$ conformado por 19 alumnos según el instrumento se encuentra en competente, y el $48 \%$ que representa a 22 alumnos se encuentran en el nivel con dificultad.

\section{Interpretación}

Por lo tanto, se puede establecer que el mayor porcentaje de la población evaluada se mantiene en el nivel con dificultad representado por el 48\%, se encuentran en este nivel por presentar omisiones, adiciones al momento de escribir. Además, un porcentaje medio representado por el $41 \%$ se establece en el nivel competente. Esta subprueba pretende indagar respecto a los procesos fonológicos de los estudiantes. Finalmente, el menor porcentaje representado por el $11 \%$ de estudiantes se sitúan en el nivel muy competente es decir la población alcanza y domina esta subprueba.

\section{Conclusiones}

- La aplicación de los instrumentos validados como: la encuesta para padres de familia y la utilización del test LEE, nos han permitido establecer la estrecha correlación entre la relación parental y el desarrollo de la Lecto-escritura puesto que se determinó que los progenitores sí influyen en el desarrollo lectoescritor en los estudiantes de la escuela de educación básica "Fe y alegría" del cantón Ambato. Debido a que los resultados estadísticos corroboraron que los padres al aplicar un plan para incentivar actividades en la lectura y escritura tienen un impacto en áreas como la lectura de palabras y comprensión de textos.

- Los resultados obtenidos resaltan la importancia que tiene la participación de los padres de familia en este tiempo de educación; debido a que actualmente juegan un papel de docente tutor sobre sus hijos., además se hace evidente la necesidad de que los padres se involucren en la educación de los hijos debido a que un gran porcentaje no forman y se mantiene al margen de la educación de sus hijos.

- Para Finalizar luego de haber aplicado el instrumento estandarizado el test Lee se evidencia que la mayor parte de la población se encuentra en el nivel "con dificultad" es decir presentan dificultades en áreas como lectura de palabras esta se caracteriza por presentar una lectura lenta, además de ser pausada reflejando errores a la hora de decodificar los grafemas, también en la sub prueba de lectura de pseudopalabras presentan dificultades en procesos sub léxicos es decir les cuesta convertir los grafemas en fonemas, finalmente la sub prueba de prosodia hace referencia a la dificultad en el proceso sintáctico de la oración y a la 
utilización de los signos de puntuación, se evidenció una lectura silábica es decir que los niños suelen leer las palabras separándolas en sílabas.

- Es necesario generar estrategias y actividades lúdicas que busquen generar un ambiente de confianza entre padres e hijos con la finalidad de que las dos partes interactúen y los padres cumplan el rol de brindar soporte y ser la guía durante este proceso para el niño, aplicando actividades innovadoras, que logren captar la atención del infante.

\section{Referencias bibliográficas}

Calle, A. M., Aguilar, M. \& Navarro, J. I. (2016). Desarrollo evolutivo de la conciencia fonológica: ¿Cómo se relaciona con la competencia lectora posterior? Revista de Investigación en logopedia, 22-41.

Carpio Brenes, M. (2017). Adquisición de la lectura experta en estudiantes costarricenses mediante las estrategias pictofónicas. Revista Electrónica Educare.

Díaz Barriga, Á. (2020). La escuela ausente, la necesidad de replantear su significado. En I. I. Educación, Educación y pandemia. Una visión académica (págs. 19-49). Coyoacán, 04510, Ciudad de México: UNAM.

Esquivel-Ancona, F. \& Aldrete-Cortez, V. (2019). Prerrequisitos para el aprendizaje académico en niños con alto y bajo rendimiento escolar. Revista de psicología y ciencias del comportamiento de la Unidad Académica de Ciencias Jurídicas y Sociales.

Gil, J. M. (2019). Lectoescrita como sistema neurocognitivo. Educación y Educadores, $422-447$.

Gutiérrez Fresneda, R. \& Díez Mediavilla, A. (2018). Conciencia fonológica y desarrollo evolutivo de la escritura en las primeras edades. Educación XX1, 395-415.

Gutiérrez, R. (2018). Habilidades favorecedoras del aprendizaje de la lectura en alumnos de 5 y 6 años. Revista signos, 45-60.

Valenciano-Canet, G. (2019). La metáfora como alternativa metodológica para investigar y reflexionar acerca de la práctica pedagógica en la enseñanza de la lectoescritura. Revista Electrónica Educare.

Ramírez-Lucas, A., Ferrando, M. \& Sainz, A. (2015). ¿Influyen los Estilos Parentales y la Inteligencia Emocional de los Padres en el Desarrollo Emocional de sus Hijos Escolarizados en 2o Ciclo de Educación Infantil? Acción Psicológica, 65-78. 
Rosero Prado, A. L. \& Mieles Barrera, M. D. (2015). Familia y lectura en la primera infancia: una estrategia para potenciar el desarrollo comunicativo, afectivo, ético y creativo de los niños. Itinerario Educativo: revista de la Facultad de Educación, 205-224.

Silva-Peña, I., Tapia, R. \& Ibáñez, M. (2016). Concepciones Docentes sobre la Escritura en Primer Año de Educación Básica. Paradigma.

Krumm, G., Vargas- Rubilar, J. \& Gullon, S. (2017). Estilos parentales y creatividad en niños escolarizados. Psicoperspectivas, 161-182.

Razeto Pavez, A. (2016). Estrategias para promover la participación de los padres en la educación de sus hijos: el potencial de la visita domiciliaria. Estudios Pedagógicos, 449-462.

Palomeque García, I. J., Alfonso Moreira, Y., \& López, M. M. (2018). La participación de la familia en la institución educativa: sus condiciones en el proceso pedagógico. Revista Universidad y Sociedad, 398-406.

Valenciano-Canet, G. (2019). La metáfora como alternativa metodológica para investigar y reflexionar acerca de la práctica pedagógica en la enseñanza de la lectoescritura. Revista Electrónica Educare.

Valencia-Echeverry, J., García-Murcia, D. C., Londoño, J. D., \& Barrera-Valencia, M. (2019). Habilidades gnósico-práxicas relacionadas con dificultades del aprendizaje de la lectura y la escritura en individuos de 9 a 12 años. CES Psicología.

Velásquez Vergara, S., \& Ruidiaz Gómez, K. (2021). La educación en tiempo de pandemia. Revista Cuidarte.

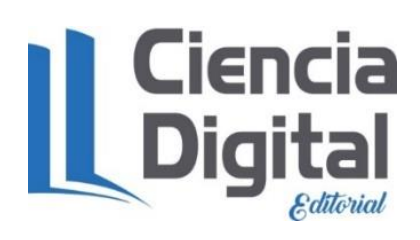


El artículo que se publica es de exclusiva responsabilidad de los autores y no necesariamente reflejan el pensamiento de la Revista Conciencia Digital.

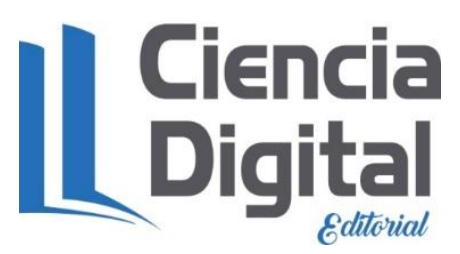

El artículo queda en propiedad de la revista y, por tanto, su publicación parcial y/o total en otro medio tiene que ser autorizado por el director de la Revista Conciencia Digital.
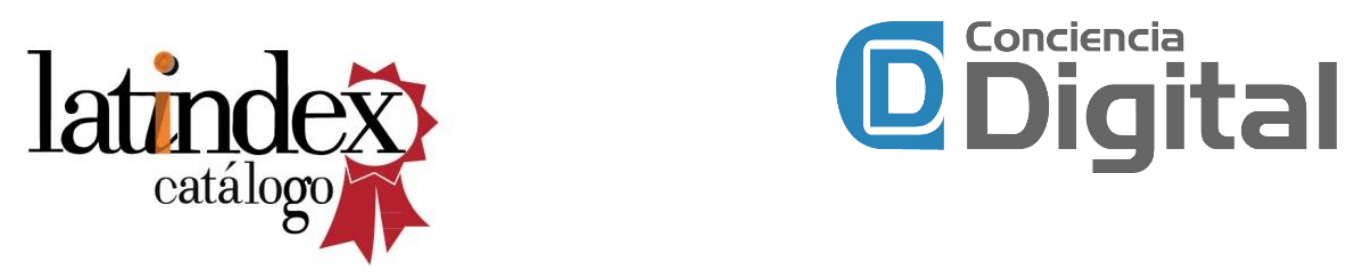

Indexaciones

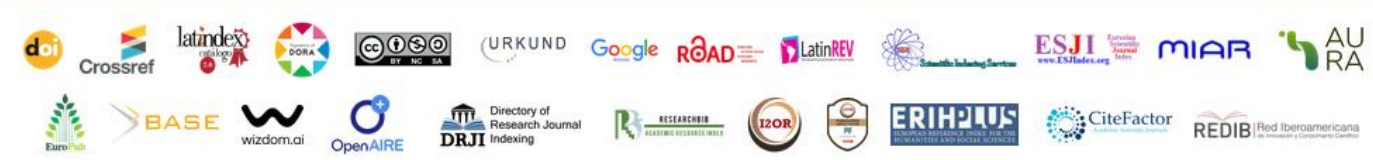

